\title{
A Study on the Relationship Between Homosexuals' Coming-Out, Self-identity, Loneliness and Perceived Social Support
}

\author{
Zheng Zhang ${ }^{1}$, Fang $\mathrm{He}^{2}$, Shiyun $\mathrm{Liu}^{3}$, Haonan Tang ${ }^{4}$, Jiaxiang $\mathrm{Li}^{5}$, Jilin Zou ${ }^{1}$, \\ ${ }^{1}$ Department of Psychology, School of Education, Linyi University, Linyi, China \\ ${ }^{2}$ Department of Accounting, School of Management, Lanzhou University, Lanzhou, China \\ ${ }^{3}$ Department of History, School of Nan Tong University, Nantong, China \\ ${ }^{4}$ Department of Psychology, School of Educational Sciences, Huaiyin Normal University, Huaian, China \\ ${ }^{5}$ Department of Psychology, School of Capital Nomal University, Beijing, China
}

Email address:

zoujl@lyu.edu.cn (Jilin Zou), hldzzz@foxmail.com (Zheng Zhang)

${ }^{*}$ Corresponding author

\section{To cite this article:}

Zheng Zhang, Fang He, Shiyun Liu, Haonan Tang, Jiaxiang Li, Jilin Zou. A Study on the Relationship Between Homosexuals' Coming-Out, Self-identity, Loneliness and Perceived Social Support. Psychology and Behavioral Sciences. Vol. 10, No. 2, 2021, pp. 63-80. doi: $10.11648 /$ j.pbs.20211002.11

Received: March 11, 2021; Accepted: March 23, 2021; Published: March 30, 2021

\begin{abstract}
Background: The long history of diversified population structure has brought the inclusiveness in Chinese culture, but in the other hand Confucian culture give birth to the conformism and moderation. The rapidly changing modern society and increasing opportunities to interact with different cultures have made the younger generation in China more open, but the overall environment has not receded to maintain and adhere to the traditional view of marriage and love, only heterosexuality is normal, has created cognitive clashes and more implicit social conflicts. In such a complex environment, homosexuals who have been outside the mainstream values for ages are apt to face more personal adaptability and mental health problems, which are more obvious in the two psychological qualities of self-identity and loneliness. Methods: We practiced mixed method to research, using perceived social support scale, loneliness scale, gay self-identity questionnaire and semi-structured interview questionnaire as tools, had measured 1962 individuals of different sexual orientation to carry on the survey, and deeply interviewed seven subjects including researchers, encode and establish models according to the collected information. Results: (1) Gay and bisexual men had significant gender main effect on self-identity, and female group had higher self-identity than male. Homosexuals' loneliness, social support and self-identity have significant residential landlord effects. The higher the level of residential area, the higher the self-identity. The higher the level of residential area, the higher the self-identity. The level of education affects the loneliness and perceived social support of individuals with different sexual tendencies (2) Perceived social support has negative correlation with loneliness in different groups the coefficient is $0.56-0.66$, the higher the perceived social support the lower the loneliness. (3) Perceived social support mediated the relationship between coming out and loneliness, gender moderated the correlation of perceived social support and loneliness. (4) The phenomenon of gender role division exists in various kinds of the non-heterosexual group, and there is no unified operational definition, which can be evaluated through multi-dimensional evaluation. (5) Gender and age have effect on the behavior of coming out, senior female are the last group to come out, exactly contrary to the young men. Certain orders and stages consist in coming-out, in a general way, self-identity is the starting point of coming-out, and the process is influenced by social support, economic conditions, family and more factors.
\end{abstract}

Keywords: Homosexual, Self-identity, Loneliness, Perceived Social Support, Coming out, Gender 


\section{Introduction}

The discussion on the psychological state of homosexuals in the context of Chinese culture is obviously different from that in Europe and America and Africa. Modern China has rapid development of economy and profound traditional culture, in the atypical balance constituted by the mainstream Confucian culture and highly open economy, homosexuals as sexual minority, is in an awkward position. Minority sexual orientation is decriminalization, negligible and has high supporting degree in the younger group, but also faces a stigmatized, and political marginalization. Negative attitude for homosexuals often is not in the study of physiology and pathology, but people from religions and social construction or hypothesis and prejudice. It can be conducted from changes from kinds of diagnostic criteria of mental disorders: in 1973 the American psychiatric association's diagnostic and statistical manual (DSM) will remove gay, but the revised version 3 (DSM - 3) includes self-discordant homosexual, a unit disease can be treated. In a subsequent revision (DSM-3-R), self-discordant homosexuals were also removed. When physiology or pathology proves that homosexuality is not a disease, the prejudice is retained and used as a weapon against homosexuals.

When gay people realize their sexual orientation, they often go through a period of shock and confusion to adapt to their identity, understand the status of this identity in the social environment, and choose whether to hide their true sexual orientation or fight for equal rights with others as a small part of the society. We can get a lot of sustaining comments for homos on the Internet, but that's not always the case. There are still some people who advocate traditional family patterns and maternity concepts, taking maternity and conventional ethics as shackles for homosexuals. Besides, the support on the virtual network is not enough to continue in real life, much less be equivalent to the physical practice. Caught between support and opposition, the mental health of gays and lesbians needs our attention.

With the opening of society, more and more attention has been paid to the research of homosexuals coming out, self-identity, loneliness and perceived social support in China. Some scholars have investigated the development of self-identity of homosexuals, and found that the development of self-identity of homosexuals has experienced four stages: homosexual attraction, self labeling, homosexual sexual contact and self disclosure, while the self-identity of lesbians has experienced five stages: Awakening stage, depression stage, conflict stage, calm stage and stable stage. Generally speaking, there are significant differences in sexual attitude, sexual behavior and self-identity between gay and lesbian. In terms of whether to choose to come out, domestic scholars have found that the common living condition of homosexuals in China at this stage is to identify but not come out, that is, to be able to accept their homosexual identity without revealing their homosexual identity to others, especially family members.

We tried to explore the differences between Chinese homosexuals and people of other sexual orientations on the correlation of social support and loneliness through both qualitative and quantitative methods, and to investigate the effect of self-identity, coming out, biological gender and gender role in the relationship between self-identity and loneliness.

\section{Review}

\subsection{Self-identity of Homosexuals}

The meaning of identity popular in modern sociology, was first given by the Austrian, psychologist Freud. It refers to the process of individuals' psychological and spiritual convergence with others, and is the initial manifestation of emotional connection with others. Self-identity, as the name implies, means that an individual's view of himself is consistent with his or her own situation and accepts all aspects of oneself. The self-identity of homosexuals means that homosexuals can be clear known of their homosexual orientation, and actively join in the homosexual group, positively organize their personal life as homosexuals, and have a positive interaction with the outside world [1]. If they cannot identify with their own homosexual identity, homosexual people are prone to undergo self-identity obstacles, affected by a series of physiological and psychological problems $[2,3]$.

There are few studies on homosexuality in China, the topic of gay self-identity gradually attracted attention after the reform and opening up. In 1989, Wang Xiaobo and Li Yinhe conducted a case study on gay men in an interview style. They then propose a blank occupancy theory of how homosexuality arises, suggesting that the first sexual encounter with someone of the same sex may fill in the gaps in sexual experience and become immobilized, ultimately leading to an individual's homosexual orientation [4]. Undoubtedly, this kind of conjecture about orientation identity has a passive, non-innate color. [5]

Biological theories about the causes of homosexuality are mainly based on three hypotheses: genetic factors, sex hormone levels and brain structure [6]. The genetic hypothesis holds that homosexuality is inherited from a recessive gene. If both partners carry the gene, their offspring may be homosexual. As for the hypothesis of sex hormone levels, some scientists have done experiments on animals to find the effect of hormones on sexual orientation, and speculated that it may also have an effect on people. The brain structure hypothesis suggests that homosexuality may be associated with regulatory activities in the hypothalamus, which is found to be smaller in homosexuals than in heterosexuals $[7,8,9]$. The three hypotheses are productive but have not been confirmed conclusively, so the biological theories about the causes of homosexuality are only conjectures at present [10].

Internet technology has a great impact on the interaction of Chinese gay communities. This change leads gay groups to rethink their position and value and strengthen some 
self-identity [11-13]. Compared with domestic researches, foreign scholars' researches on homosexual self-identity are more systematic. In 1984, Cass proposed the six-stage theory of gay self-identity: 1. Identity Confusion 2. Identity comparison 3. Identity tolerance 4. Identity Acceptance 5. Identity pride 6. Identity Synthesis [14]. According to Cass, the process of gay identity is the process of finding out the nature of one's sexual orientation, and this kind of identity is hard to change once it is complete for lifelong time. After the APA removed homosexuality from the list of mental illnesses in 1973, prejudice against the gay community gradually decreased, and the focus of research on homosexuality gradually shifted from pathology to sexual identity. The study found that gay people who actively identify with their sexuality have a healthier psychological environment, more likely to deal with the physical and psychological problems associated with their sexual minority orientation, and more positive to interact with society [15]. Homosexual orientation self-identity plays an important role in the physical and mental health of homosexuals.

\subsection{Coming out -- The Disclosure of Sexual Orientation}

The word coming out was meant to reveal the truth rather than keep secrets. When used to express sexual orientation, it generally refers to the behavior of sexual minority groups such as gay, lesbian or bisexual to disclose their sexual orientation. Generally speaking, the coming out of the closet of sexual minorities means that they have accepted their sexual identity and integrated this identity into their own life experience, which is the most significant manifestation of gay self-identity [16]. But in daily life, not all individuals who have a favorable sense of identity with their sexuality choose to come out. That is, the final result of self-identity is not necessarily coming out, so it cannot be used to directly measure the status of self-identity of homosexuals [17].

In previous studies, self-identity of homosexuals was considered as the starting point of coming out [18]. Dank first put forward the stage theory of gay coming out, he found that there is usually six years' time between the homosexuality for the first time and self definition as gay, so he proposed identity phase theory, including redefined gay stereotypes, gay and lesbian community identity and self-acceptance and the diclosure [19]. Later, on the basis of summarizing previous studies, Richard Troiden puts forward a four-stage model of gay identity formation: 1 . Sensitization 2 . Identity confusion 3. Identity hypothesis 4. Participation [20]. Plummer proposed that after getting rid of the confusion about one's sexual orientation, building self-confidence and realizing self-identity, there are generally three forms and purposes to come out to others: 1. Private coming out 2 . Public coming out 3. Coming out on political grounds [21].

Recent years, there have been many new advances in the theoretical research on homosexual identity and coming out. For example, Yoshida proposed the dynamic pendulum model of identity, believing that in the process of identity reconstruction after severe trauma, the formation of identity concept swings back and forth like a pendulum [22].
Scholars study the situation of gay people coming out of the closet from many perspectives. From the perspective of cultural diversity, some scholars have textual research on the differences of homosexual identity among different ethnic groups [23]. Some scholars have also analyzed the issue of sexual minorities coming out in the analysis framework of physical and mental health [24]. Others take the issue of coming out of the as a starting point to discuss how changes in the legal environment affect the living conditions of sexual minorities $[25,26]$.

So far, there have been few studies on gay coming out in China, but some scholars have noticed the difficulties of gay men coming out in China. Wang Qingfeng believes that Chinese gay men generally live in a state of identity without coming out. Even gay men who have a good identity are still reluctant to reveal their homosexuality to people around them, especially to their parents, and many gay men are struggling in the state of marriage exist in name only [17]. Fu Xiaoxing also noted that gay men generally believe that concealed sexual orientation equals repressed individual desire, but due to fear that revealing their identity will lead to extreme disappointment or anger of parents, family is still a difficult barrier for homosexual identity disclosure [27]. Wei Wei found that the vast majority of interviewees did not think that openness of sexual identity among gay people belongs to coming out of the closet [28]. Feng Jian and Zhao Nan pointed out that homosexual social network is separated from the daily life space, that is, the expression of sexual identity in the network does not mean that individuals integrate homosexual sexual identity [29].

Some studies have found that sexual minorities stress model considers coming out can reduce stress, promote self-cognition and thus improve their own traits [30]. Empirical studies have provided support for this, namely coming out as a protective factor can reduce the risk of suicide of homosexuals [31]. Empirical studies also show that coming out of the closet, as a protective factor, can promote the mental health of Chinese homosexuals [32, 33].

As mentioned above, there is not a sufficient and necessary relationship between coming out of the closet and the self-identity of homosexuals, so the reasons why homosexuals choose to come out are worthy of further exploration. In this study, we suggest that, in addition to self-identity, whether gay people choose to come out may be related to feelings of loneliness and perceived social support.

\subsection{Loneliness}

Loneliness is a subjective psychological experience or feeling, which mainly occurs when individuals feel the lack of satisfactory interpersonal relationship, and there is a gap between their desire for communication and the actual level. Peplau [34] put forward that loneliness is a kind of unpleasant experience when social relations network is insufficient, including the insufficient quantity and low quality. In recent decades, there has been a lot of research on loneliness and a lot of achievements have been made. Studies have shown that loneliness is closely related to social support 
and mental health. Therefore, the research on loneliness is not only limited to the field of social relations, but also an important topic in the field of mental health and personality psychology. [35, 36]

Loneliness is a painful and unpleasant subjective experience or psychological feeling from the defect of interpersonal relationship. It may be related to the individual's environment, but it does not constitute a causal relationship [37]. Cross-cultural studies on loneliness show that loneliness is significantly correlated with physiological and psychological factors such as mobile phone addiction, sleep quality and self-esteem, and is inversely proportional to social support, intimate relationship quality and degree of self-identity. Nowadays, people show more and more symptoms of depression and anxiety, and a large number of subjects report that they are in a state of long-term psychological sub-health and lack of deep emotional connection. Homosexual orientation has been outside the social mainstream orientation for a long time, so it has become a group more susceptible to loneliness. The research and analysis of the causes and effects of loneliness is of great significance to the field of mental health and personality development. [38]

For the homosexual community, the difficulty to understand their special status in the social environment, hardness to establish a stable and long-term intimate relationship due to the small number of similar people, as well as the understanding and support from family and friends and other problems or not, which all cause emotional loneliness to a certain extent [39]. However, the real discrimination, prejudice, stereotype and the fear that they will be isolated by other social groups also lead to more homosexuals choosing to hide their authenticity and avoid the deep emotional communication with others, so that they are in a long-term social loneliness. [40, 41]

The spread of the Internet and the growth of social media have changed the way the gay community interacts. The emergence of more and more social campaigns such as sex education, gay community destigmatization and same-sex marriage legislative initiatives have played an important role in establishing a good self-identity and alleviating social loneliness among homosexuals [42]. But at the same time, the ethical and moral condemnation under the traditional view of marriage, love and procreation is also widespread, which not only makes homosexuals face difficulties in the voice of both sides, but also causes the public to think deeply about marriage and love, family, offspring rearing, social and cultural tolerance [43]. Therefore, it has more important social significance to pay attention to the actual living conditions and mental health of homosexuals from the perspective of loneliness. [44, 45]

\subsection{Comprehension of Social Support}

Social support means that individuals receive spiritual and material help from all aspects of society (such as family, friends, teachers, etc.) in their own social network, including direct help and potential help. It is a kind of social behavior with positive significance. Understanding social support is the individual's feelings and beliefs about the above helping behaviors. There is a certain correlation between the two. In real life, perceiving social support has a greater effect on people's psychology than the actual social support [48].

A large number of scholars have jointly shown that the stronger the social support network behind an individual, the easier it is to cope with challenges from various aspects. Social workers believe that by changing an individual's social network, they can make up for the lack of personal resources and improve their resilience. The sources of control affect perceived social support, especially through their indirect effects on problem solving and subjective well-being. In addition, past situations are more likely to influence college students' perception of social support than current situations. [46] Generally speaking, women perceive social support better than men [47].

Social support, as a positive psychological feeling, is positively correlated with mental health level, life satisfaction, subjective well-being, self-esteem, and positive emotions, and negatively correlated with loneliness, depression, anxiety, online bullying, and negative emotions [49-58]. Some scholars believe that higher understanding of social support can make individuals acquire more optimistic and confident adaptation mode, thus producing more positive psychological state. However, it does not mean that social support is beneficial to all, and some researchers believe that too much social support may have an impact on individuals' self-decisions and social relationships [59, 60].

Social support is of extreme significance for gays and lesbians, gay people are faced with so many psychological problems, not because of sexual orientation for physical or psychological harm brought by itself, but rather in self cognition and the contradiction between social cognition, make the individual not be able to smoothly integrated into the social structure [61]. When homosexuals receive enough social support, they will also optimize their self-identity, so as to realize a compatible self-cognition structure and promote the development and integrity of their personality [62].

However, a distinction should be made between actual social support and perceived ones [63]. For example, sometimes parents who are not friendly to homosexuals may still show their love for their children and give no support for their children's marital problems but their children still feel positive emotions. It's a conflict between kinship warmth and cognitive bias [64]. On the Internet, we may see some votes or micro-blogs expressing support for homosexuals, but there is often a gap between online support and real support, let alone real action in life. Even though what we see may not be an individual's true implicit attitudes, we make judgments about how much social support we feel based on behavior and explicit attitudes [65-68]. Therefore, we focus not on describing the actual social support received by homosexuals, but more on the subjective feelings of homosexuals 


\section{Research Contents}

\subsection{Research Objects}

A total of 2356 subjects were randomly selected from provinces, autonomous regions, Hong Kong, Macao, Taiwan, Japan, and the United States by random sampling method. After screening and verification, the effective sample size was
1962 , the effective rate was $83.27 \%$, and the average age of the subjects was $22.79 \pm 5.59$ years old. In terms of residence, most of them live in cities, while a small number live in towns or villages. In terms of education level, most of them have a bachelor's degree, while the rest are scattered. Therefore, the main objects of this study are urban residents with different sexual orientation who have received higher education.

Table 1. Number of samples of gender and sexual orientation.

\begin{tabular}{llllll}
\hline & Homosexual & heterosexuality & bisexual & Other sexual orientation \\
\hline Male & 580 & 143 & 72 & 10 & \\
Femal & 359 & 416 & 328 & 48 & 105 \\
Other gender & - & - & 1 & 5 & 6 \\
sum & 939 & 559 & 401 & 63 & 1151 \\
\hline
\end{tabular}

\subsection{Research Tool}

\subsubsection{Gay Self-Identity Scale}

This study adopts the Chinese revised version of the Gay Self-Identity Scale compiled by Dan Qiao from Nanjing Normal University, with a total number of 25 items. The Likert 6-type subscale is adopted. The higher the score, the stronger the self-identity. The scale is divided into six factors, which are identity recognition, acceptance of attention, identity uncertainty, identity superiority, process difficulty and identity centrality. The reliability and validity of the scale were tested. The internal consistency reliability ranged from 0.610 to 0.799 , and the Cronbach coefficient of the total self-identity questionnaire was 0.749 . The total reliability of retesing is 0.882 , the score of each dimension is between 0.625 and 0.883 , and the content validity and structure validity are good. It is culturally adaptable in China and suitable for relevant research. In this study, the Colombach coefficient of the scale was 0.771 .

\subsubsection{Comprehension Social Support Scale}

Compared with other social support scales, the perceiving social support scale puts more emphasis on the social support that individuals feel themselves. It is used to measure the social support that individuals feel from various sources, such as family, friends and other people. Meanwhile, the total score can reflect the total degree of social support. The scale has a total of 12 items and uses Likert scale 7-level scoring method. The higher the score, the stronger the perceived social support. The Cronbach coefficient is between 0.85 and 0.91 , and the retest reliability is between 0.72 and 0.85 , which is reasonable in reliability and validity. Many scholars in China have used this scale for research, showing its adaptability to Chinese culture. The Cronbach coefficient of the scale in this study was 0.899 .

\subsubsection{UCLA Loneliness Scale}

The simplified version of UCLA Loneliness Scale includes 6 items, all of which are positive scoring items. The scale uses a 4-point Likert scale, with higher scores associated with greater loneliness. The Cronbach coefficient of the scale in different populations was 0.83 , and the retest reliability was
0.715-0.90. Good reliability and validity, moderate number of questions, in line with the psychometrics standards, suitable for the measurement of loneliness. In this study, the Colombach coefficient of the scale was 0.839 .

\subsubsection{Semi-structured Interview Questionnaire for Homosexuals}

According to the needs of the research topic, the team members integrated six dimensions, including identity, emotional experience, coming out of the closet, marriage expectation, discrimination/support perception, development, and about 30 questions. In the interview process, the statements of questionnaire questions and the questions with inappropriate or low response rate were deleted and corrected successively.

\subsection{Research Methods}

The questionnaire is designed through the online questionnaire website and distributed to the gay community through special organizations (such as PFLAG, GaySpot and other gay rights organizations). The rest of the questionnaire is distributed to the general public through social media channels such as Weibo, WeChat and QQ. For non-heterosexuals, the questionnaire included demographic variables, self-identity, perceived social support, and loneliness. In addition, gender roles and proactivity in relationships were measured, but not as a stable variable. For heterosexuals, questionnaire information included demographic variables, perceived social support, and loneliness

In addition, we through semi-structured interview includes scale on several gay in-depth interviews, interviews, interviewed by telephone or face-to-face interviews, in the form of unified instructions, read the informed consent and confirmed after the interview, interview recordings, all sorts through conversation immediately after the interview. The interviewees are coded, and the codes and information are as follows. The first number is the number of the interviewee, the second number is the gender of the interviewee (male is 1 , female is 2 , other gender is 3 ), and the third number is the number of the interviewee corresponding to an interviewer.

Except real-name interview information, researchers also capture the answers of specific questions on Zhihu and other 
websites, quote the answers on the basis of ensuring random information, and anonymously code the answer authors.
Network information is only used to support the conclusions that have been drawn, but not for extraction and analysis.

Table 2. Coding explanation of semi-structured interviewees.

\begin{tabular}{llll}
\hline alias & code & Sexual orientation & Personal information \\
\hline Yiyi & 121 & bisexual & 20, University degree, 0.5 \\
Erdan & 211 & homosexual & University degree, 0.5 (Gender roles are in the middle) \\
Xiaohong & 222 & bisexual & University degree, No attributes \\
Jiuhun & 321 & homosexual & 19, junior degree, P \\
Baishu & 322 & homosexual & 23, University degree, 0.5 \\
Ajiao & 323 & heterosexual & 20, University degree \\
Ahai & 411 & homosexual & 22 , University degree, 0.5 \\
Lulu & 412 & homosexual & 23, University degree, 0.5 \\
\hline
\end{tabular}

\subsection{Data Processing}

Use SPSS23.0, the Process plugin, NVIVO11, and the SPSSAU online data processing tool.

\subsection{Research Hypothesis}

Due to the large number of variables involved in the study, we proposed the following hypotheses regarding the relationship and mutual influence among the variables:

Sexual minorities with good self-identity can effectively reduce loneliness and perceive more social support.

Due to the influence of traditional culture, social morality, stereotype and other factors, we speculate that there is a certain degree of gender difference in understanding social support, and this gender factor will interact with the self-identity of sexual minorities and jointly affect their status of coming out of the closet.

according to the cognitive dissonance Festinger lattice theory, we think for a long time to hide, to hide the true inner self will cause sexual minorities in a certain degree of cognitive dissonance, lead to the huge psychological pressure and loneliness, in order to reduce the pressure to complete self-identification, sexual minorities tend to try out, partly out of the ark. Therefore, we hypothesized that self-identity had an effect on coming out of the closet, and that the outcome of coming out would in turn affect their self-identity.

\section{The Result}

\subsection{Quantitative Research Result}

\subsubsection{Variables Descriptive Statistics Analysis}

To obtain the status of all the participants' self-identity, loneliness and perceived social support, We conducted ANOVA: The gender main effect of homosexual self-identity was significant $(\mathrm{F} \geq 3.879, \mathrm{P}<0.05)$, and that of bisexual self-identity was significant $(\mathrm{F}=4.60, \mathrm{P}=0.011)$, and self-identity of female group was higher than male group. The residence main effect of loneliness, comprehension of social support and self-identity were significant for homosexuals $(\mathrm{F} \geq 3.99, \mathrm{P}<0.019)$. And the higher the level of place of residence, the higher the self-identity. None of main effect of loneliness, social support and self-identity was significant for bisexuals and other sexual orientations. The main effect of educated levels on loneliness, perceived social support and self-identity was significant $(\mathrm{F} \geq 2.716, \mathrm{P}<0.04)$ for homosexuals, and the main effect of educated levels on loneliness and perceived social support was significant for heterosexuals $(\mathrm{F} \geq 5.273, \mathrm{P}<0.001)$. The main effect of educated levels on comprehension social support was significant for bisexuals $(\mathrm{F}=5.905, \mathrm{P}=0.001)$.

Table 3. The mean value and standard deviation of each variable ( $n=1962)$.

\begin{tabular}{llllll}
\hline Biological gender & Sex orientation & Self-identity & loneliness & Perceived social support & Status of coming out \\
\hline Male & Homosexual & $91.03 \pm 13.22$ & $14.98 \pm 3.87$ & $57.42 \pm 10.59$ & $2.27 \pm 0.78$ \\
& Heterosexual & & $13.39 \pm 3.29$ & $60.96 \pm 10.76$ & \\
& Bisexual & $80.86 \pm 13.67$ & $14.07 \pm 3.86$ & $58.26 \pm 10.05$ & $1.83 \pm 0.78$ \\
& Other & $82.90 \pm 9.96$ & $16.50 \pm 3.171$ & $62.00 \pm 9.90$ & $1.99 \pm 0.99$ \\
& Totals & $89.80 \pm 13.61$ & $14.63 \pm 3.81$ & $58.18 \pm 10.64$ & $2.22 \pm 0.79$ \\
Female & Homosexual & $93.09 \pm 12.45$ & $13.99 \pm 3.82$ & $55.97 \pm 11.49$ & $2.24 \pm 0.7$ \\
& Heterosexual & & $13.79 \pm 3.28$ & $62.35 \pm 10.98$ & $1.98 \pm 0.78$ \\
& Bisexual & $85.99 \pm 13.15$ & $14.02 \pm 3.63$ & $59.22 \pm 10.07$ & $1.83 \pm 0.72$ \\
& Other & $86.88 \pm 14.22$ & $14.54 \pm 3.98$ & $55.83 \pm 11.33$ & $2.10 \pm 0.75$ \\
& Totals & $89.51 \pm 13.33$ & $13.95 \pm 3.58$ & $59.20 \pm 11.22$ & 5.00 \\
& Bisexual & 93.00 & 14.00 & 60.00 & $2.6 \pm 1.67$ \\
& Other & $94.20 \pm 10.57$ & $16.60 \pm 2.70$ & $52.40 \pm 4.67$ & $3.00 \pm 1.79$ \\
\hline
\end{tabular}




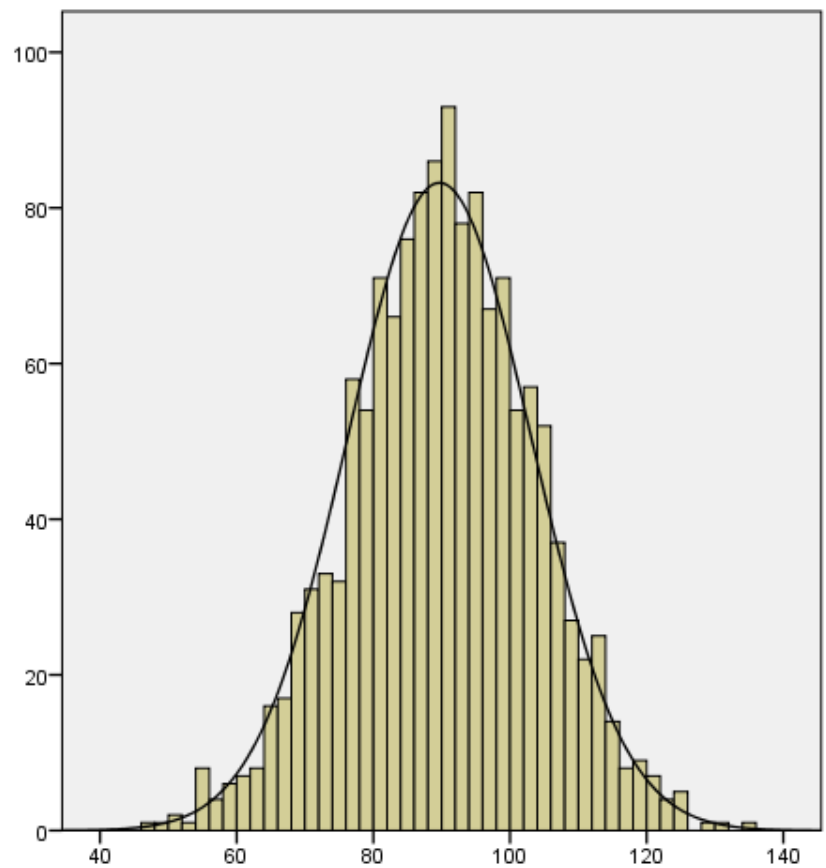

Figure 1. Distribution of self-identity scores of non-heterosexuals.

\subsubsection{Correlation Analysis and Path Analysis of Each Variable}

Self-identity of homosexuality and bisexuality group and loneliness appear low negative correlation $(\mathrm{p}<0.01)$, self-identity and perceived social support appear low positive correlation $(\mathrm{p}<0.01)$, loneliness showed high negative correlation with the understanding of social support, and that now illustrates that the domestic sexual minority group in the case of good self-identity can reduce loneliness and feel more social support, the reason may be the current domestic mainstream still cannot treat sexual minorities as ordinary people completely, but has been great progress than in the past. There was a higher negative correlation between loneliness and perceived social support in the heterosexual group ( $\mathrm{P}<0.01)$, but the correlation was still slightly smaller than that between loneliness and perceived social support in the homosexual and bisexual group, suggesting that the LGBT group often needs social support more than the heterosexuals.

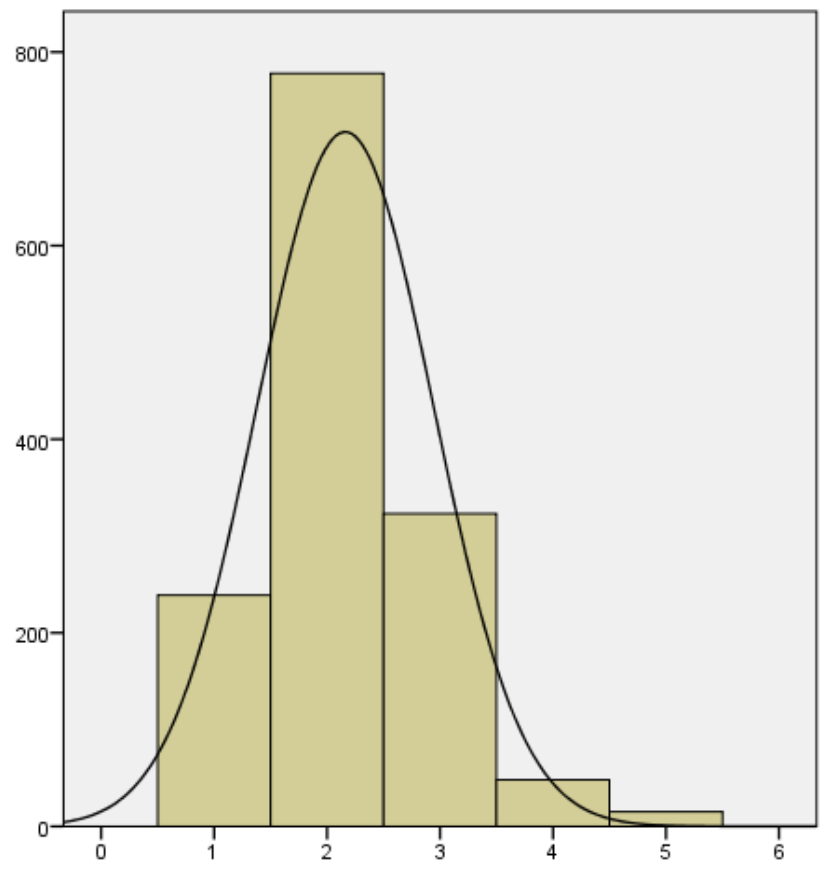

Figure 2. Distribution of coming out of the closet of non-heterosexuals.

Table 4. The descriptive statistics and correlation analysis result of each variable (r).

\begin{tabular}{|c|c|c|c|}
\hline Sex orientation & & Loneliness & Perceived social support \\
\hline & Self-identity & $-0.26^{* *}$ & $0.18^{* *}$ \\
\hline Homosexual & Loneliness & & $-0.60^{* *}$ \\
\hline Heterosexual & Loneliness & & $-0.56^{* *}$ \\
\hline & Self-identity & $-0.28^{* *}$ & $0.23^{* *}$ \\
\hline Bisexual & Loneliness & & $-0.66^{* *}$ \\
\hline
\end{tabular}

$* * \mathrm{P}<0.01$

Each dimension of identity scale is significantly correlative with total table $(\mathrm{p}<0.01)$. According to the Table 5 in addition to Acceptance Concerns and Identity Superiority Acceptance Concerns and Identity Centrality,
Identity Uncertainty and Identity Superiority, Identity Affirmation and Identity Centrality did not achieve statistical significance $(\mathrm{p}>0.05)$, every two variables, including each variables with total tables achieve statistical significance.

Table 5. The descriptive statistics results and correlation analysis of subscale and total scale of Self-Identity (r).

\begin{tabular}{lllllll}
\hline Dimension & $\mathbf{X} \pm \mathbf{s}$ & $\mathbf{1}$ & $\mathbf{2}$ & $\mathbf{3}$ & $\mathbf{4}$ \\
\hline 1 Acceptance Concerns & $19.6 \pm 5.2$ & 1 & & & \\
2 Identity Uncertainty & $13.4 \pm 3.9$ & $0.29^{* *}$ & 1 & & \\
3 Identity Affirmation & $26.1 \pm 5.8$ & $0.21^{* *}$ & $0.27^{* *}$ & 1 & & \\
4 Identity Superiority & $10.4 \pm 2.9$ & 0.01 & 0.03 & $0.23^{* *}$ & 1 & $0.09^{* *}$ \\
5 Identity Centrality & $9.8 \pm 2.4$ & 0.02 & $0.13^{* *}$ & 0.01 & 1 \\
6 Difficult Process & $10.1 \pm 3.9$ & $0.40^{* *}$ & $0.35^{* *}$ & $0.15^{* *}$ & $0.06^{*}$ & $-0.08^{* *}$ \\
7 Totals of Self-identity & $89 . \pm 13.7$ & $0.67^{* *}$ & $0.63^{* *}$ & $0.67^{* *}$ & $0.35^{* *}$ & $0.21^{* *}$ \\
\hline
\end{tabular}

$* * \mathrm{p}<0.01 * * *, \mathrm{p}<0.001$ 
All subscales in the Perceived social support Scale are significantly related to the total scale $(p<0.01)$. There is a high correlation between family support, friend support, and other support, indicating that people who can feel more support in a certain group o in their lives can often feel corresponding support from other people around them. In other words, people who do not feel support in one aspect of family, friends, or others often do not feel support in others.

Table 6. Descriptive statistical results and correlation analysis of the subscales and total scale of each dimension of perceived social support (r).

\begin{tabular}{|c|c|c|c|c|}
\hline & $\mathbf{X} \pm \mathbf{s}$ & Family support & Friend support & Other support \\
\hline Family support & $16.6 \pm 6.0$ & 1 & & \\
\hline Friend support & $20.6 \pm 4.8$ & $0.49^{* *}$ & 1 & \\
\hline Other support & $20.3 \pm 4.9$ & $0.66^{* *}$ & $0.80^{* *}$ & 1 \\
\hline Totals & $57.4 \pm 13.7$ & $0.85^{* *}$ & $0.85^{* *}$ & $0.93^{* *}$ \\
\hline
\end{tabular}

$* * \mathrm{P}<0.01$

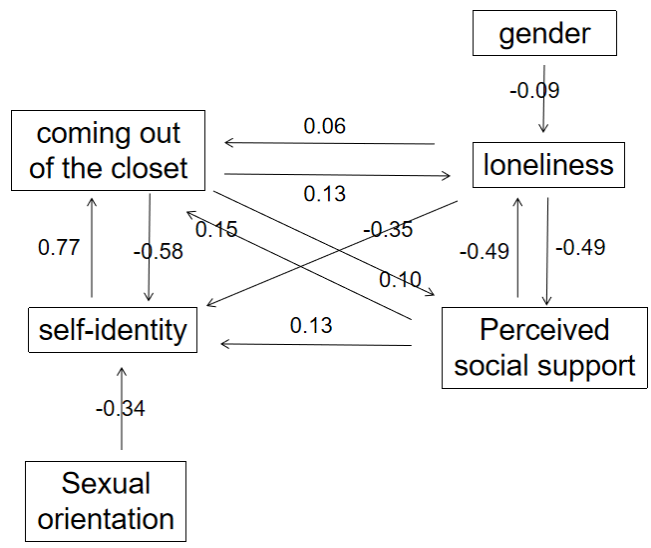

Figure 3. The path graph model of the regression coefficient of each variable.

\subsubsection{The Mediating Role of Perceived Social Support Between Coming Out and Loneliness}

According to the research hypothesis and Table 8, loneliness is significantly correlated with perceived social support $(r=-0.6, p<0.01)$, and loneliness is significantly correlated with coming out status, perceived social support is significantly correlated with coming out status. The intermediary model fits well $\left(\mathrm{R}^{2}=0.24\right)$. The direct path coefficients of the mediating effect model are all significant. In this study, 1000 Bootstrap tests were conducted and found that the mediation test of perceived social support for the influence of coming out of the closet on loneliness, the $95 \%$ interval does not include the number $0 \quad(95 \%$ CI: $-0.406 \sim 0.162$ ). Therefore, it is understood that social support has an intermediary effect when the status of coming out has an effect on loneliness. In addition, after controlling the intermediary variables, perceived social support, the independent variable coming out of the closet, still had a significant impact on the dependent variable loneliness $(p<0.05)$. Therefore, states that social support plays a part of the mediating role between coming out and loneliness.

Table 7. Mediation Effect Model Test $(n=1000)$.

\begin{tabular}{llllll}
\hline Effect & Boot SE & BootLLCI & BootULCI & z & p \\
\hline-0.29 & 0.06 & -0.41 & -0.16 & -4.46 & 0 \\
\hline
\end{tabular}

Note: BootLLCI refers to the lower limit of the $95 \%$ interval of Bootstrap sampling, and BootULCI refers to the upper limit of the $95 \%$ interval of Bootstrap sampling.

Table 8. The mediating effect analysis of comprehension of social support between coming out and loneliness.

\begin{tabular}{llll}
\hline & Perceived social support & Loneliness & Loneliness \\
\hline constant & $53.94\left(64.27^{* * *}\right)$ & $15.02\left(50.01^{* * *}\right)$ & $24.43\left(46.90^{* * *}\right)$ \\
Status of coming-out & $1.636\left(4.48^{* * *}\right)$ & $-0.26\left(-1.99^{*}\right)$ & $0.03(0.22)$ \\
Social support & & & $-0.175\left(-20.914^{* * *}\right)$ \\
$\mathrm{R}^{2}$ & 0.01 & 0.01 & 0.24 \\
Adjusted R & 0.01 & 0.01 & 0.24 \\
F value & $\mathrm{F}(1,1401)=20.05^{* * *}$ & $\mathrm{~F}(1,1401)=3.95^{*}$ & $\mathrm{~F}(2,1400)=221.29^{* * *}$ \\
\hline
\end{tabular}

$* \mathrm{p}<0.05, * * \mathrm{p}<0.01 * * *, \mathrm{p}<0.001$

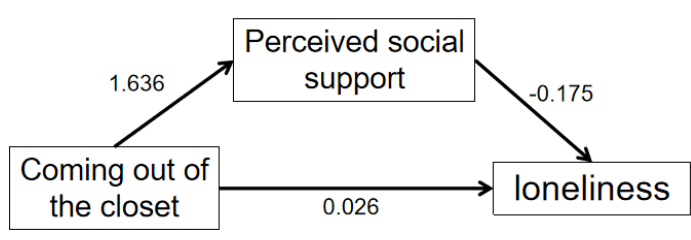

Figure 4. The mediating effect model of comprehension of social support between coming out and loneliness.

\subsubsection{The Impact of Perceived Social Support on Loneliness: The Moderating Role of Gender}

Gender still has a negative predictive effect on loneliness $(\beta=-0.483, \mathrm{t}=-3.288, \mathrm{p}<0.005)$, indicating that gender not only directly affects loneliness, but also indirectly affects loneliness through perceived social support. The direct and indirect effects of gender on social support are -0.483 and 0.05 respectively; in addition, the interaction term of gender and perceived social support has a significant negative predictive effect on the loneliness of homosexuals $(\beta=0.027$, $\mathrm{t}=1.977, \mathrm{p}<0.05$ ), so the hypothesis is tenable, that is, gender modulates the influence of perceived social support on loneliness.

To further explore the moderating role of gender in the impact of perceived social support on loneliness, we conducted 
a simple slope analysis (Table 9). The simple slope test showed that the effect of increased perceived social support on loneliness of gay men $(Z=1)(\beta$ simple $=-0.147, t=-15.859, p=0)$ compared with lesbians $(Z=-1)$. The impact of increased perception of social support on loneliness $(\beta$ simple $=-0.174$, $\mathrm{t}=-17.876, \mathrm{p}=0)$ did not change significantly.

Table 9. Simple slope analysis.

\begin{tabular}{llllll}
\hline Moderating variable levels & Regression coefficient & Standard error & t & p & 95\%CI \\
\hline Average value & -0.16 & 0.01 & -24.09 & 0 & -0.173 \\
High-level (1SD) & -0.147 & 0.01 & -15.86 & 0 & -0.147 \\
Low-level (-1SD) & -0.174 & 0.01 & -17.88 & -0.165 & 0 \\
\hline
\end{tabular}

Table 10. Analysis results of loneliness moderating effect.

\begin{tabular}{llll}
\hline & Model 1 & Model 2 & Model 3 \\
\hline Constant & $14.24\left(194.38^{* * *}\right)$ & $14.24\left(194.88^{* * *}\right)$ & $14.231\left(194.77^{* * *}\right)$ \\
Social support & $-0.16\left(-24.10^{* * *}\right)$ & $-0.16\left(-24.00^{* * *}\right)$ & $-0.16\left(-24.09^{* * *}\right)$ \\
Gender & & $-0.49\left(-3.34^{* * *}\right)$ & $-0.48\left(-3.29^{* *}\right)$ \\
Interaction term & 0.23 & & $0.03\left(1.98^{*}\right)$ \\
$\mathrm{R}^{2}$ & $\mathrm{~F}(1,1960)=580.53^{* * *}$ & 0.23 & 0.23 \\
$\mathrm{~F}$ value & 0.23 & $\mathrm{~F}(2,1959)=297.37^{* * *}$ & $\mathrm{~F}(3,1958)=199.84^{* * *}$ \\
$\Delta \mathrm{R}^{2}$ & $\mathrm{~F}(1,1960)=580.53^{* * *}$ & 0.01 & 0.01 \\
$\Delta \mathrm{F}$ value & $\mathrm{F}(1,1959)=11.18^{* * *}$ & $\mathrm{~F}(1,1958)=3.91^{*}$ \\
\hline
\end{tabular}

$* \mathrm{p}<0.05 * * \mathrm{p}<0.01 * * * \mathrm{p}<0.001, \mathrm{t}$ value in brackets

\subsection{Qualitative Research Results}

\subsubsection{Balances Between "0" and "1" -- the Label of Void and Truth}

In homosexuals, a second division is often made between two of the same sex -- that is, the gender roles within homosexuals. Among gay men, according to the degree of initiative in the sexual relationship and intimate relationship, they are divided into the active and the passive, which are called attack and accept in the Chinese context, and are also called 1 and 0 according to the organ images used in the sexual relationship between the two, of which the former represents the penis and the latter the anus. Similarly, there is a $T / P$ divide among lesbians, where $\mathrm{T}$ stands for masculine individuals and $\mathrm{P}$ for feminine individuals. Before the interview, we discussed and searched literature on the division of gender roles in homosexuality, but no positive and consistent conclusions were reached. We collected part of the comments on the network platform and put forward two hypotheses based on them:

Gender roles in homosexuality have become an indispensable part of personal information on many gay social media, and are widely used in real life. This kind of positioning or labeling is conducive to reducing the social cost, adapting to the role identity more quickly, and thus promoting self-identity. Therefore, it is reasonable to classify homosexuals as gender roles.

The rationality mentioned above is only the construction of sexual relations and intimacy by heterosexuals or homosexuals influenced by heterosexual culture, which is invisibly shaped by mainstream culture. It has limited significance to the gay community itself, and is sometimes easy to form misunderstandings and stereotypes.

Interviewees seem to be divided into different camps as to the necessity and reason of gender role division. Supporters 121 hold that it is slightly necessary, and it is necessary to use it on some dating platforms to facilitate those zeros to date ones. It's important to note that if you're looking for someone on a dating platform for sexual purposes, there's no need to draw such a fine line in your daily life. 211 said, I think it is also necessary, if the bed statement can be clear in advance, to reduce social costs, especially for men with high congenital requirements, some people can only do 1 or 0.

Opponent 222 said: But I think I have asked some of my friends and they are not strictly divided. Most of the time, people still want to have a fixed perception of the world, and they will pursue this binary division. I myself sometimes than a lot of boys just, also have a lot of time is simply weak, how can this divide? Most people have a lot of facets.

Neutral 412 says, I think it's all about how to say, for the sake of labeling, that you don't support it, that you don't like people to label you. OK. Then I can not call you, I don't think you are a zero. So 1, this is all fine. But I think when it's normal, when I know you, it's a lot easier for me to have a label.

In view of the contradiction between the above assumptions and viewpoints, we dissected and analyzed the interview text, and obtained the following gender role construction models from the dimensions of sexual relationship, economic relationship, decision-making, personality and self-assessment.

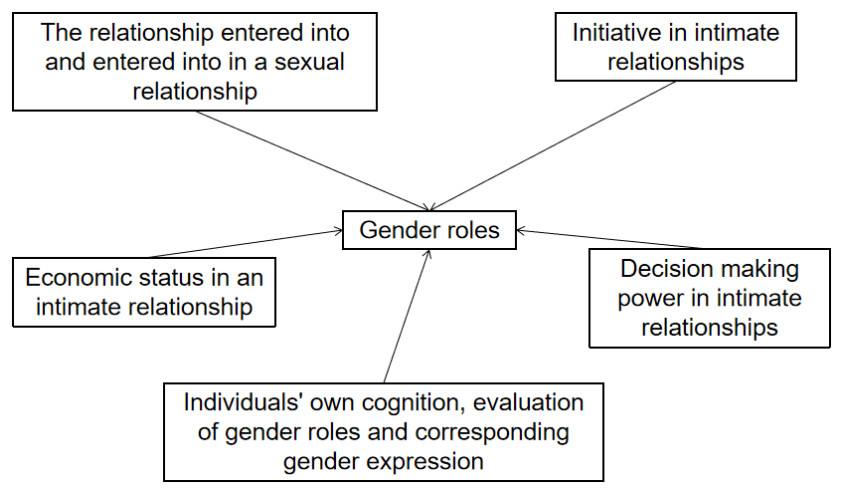

Figure 5. Model of gender role construction. 
What we're looking at is a complex model of how human emotions interact with social morality, but there are people who don't think so. There are so many dimensions to gender that we may not be able to complete the finer, more precise delineation of gender in this lifetime. When we proposed to add a gender role dimension to the study, my consideration was that it is indeed a relatively skewed pattern in intimate relationships, not a state of perfect balance, but more like a dynamic balance. Kinsey once put forward the theory of gender flow, believing that people's cognition of their own gender is not unchanged, but adjusted according to environmental changes and their own knowledge, experience, emotion and so on. This theory has since been extended to the study of sexual orientation: people's preference for a gender is also subject to changes in their environment and perception of themselves. And if we can accept the flow of gender and sexual orientation, why can't we extend it to gender roles? The more vocal opposition to gender roles is that it's a label: We've removed the gender label, now should we even label homosexuals? But all labels may be illusory, and in some ways, we use labels to serve our purpose of building better social and even intimate relationships. When gender roles just flow to a matching fit, it is so subtle and indescribable in this moment.

\subsubsection{Age and Gender -- True Moral Kidnapping}

There is often a bias in the existing social value system that older people are more respectable and younger people are frivolous and ignorant. The male is calm and reliable, the female is gentle and clever. Even more frightening is the attitude of social groups towards different groups of people making mistakes in sexual and intimate relationships. For older people, it's called old without respect. For younger people, it's Who didn't make mistakes when they were young? Men are more tolerant and forgiving; women are more forgiving. This social attitude has some historical factors. China has a long feudal society dominated by natural economy and formed a complete patriarchal system. The huge family structure gives the elderly important rights and respect, and the logic of this respect comes from the fact that the elderly's behavior is closer to and more in compliance with morality. Therefore, in the era when the concept of rule of law is not complete, the elderly, as the embodiment of justice and fairness, tend to be more fierce and sharp when their moral image is damaged. On the gender dimension, feudal culture honored men, and this inertia is still showing up even now. In ancient times, some men regarded some women as objects, that is, reproductive tools without independent personality. Therefore, in the face of such immoral things as cheating, under the feudal education, some men just think it is buying the wrong thing, while women think it is betrayal.

In view of the characteristics of the age and gender of the coming out of the gay, the author conducted a supplementary survey. Through stratified random sampling on Blued and Lesdo platforms, the author selected the gender ratio $1: 1$ and the age class according to the proportion of Chinese population yearbook, and made the line chart as follows:

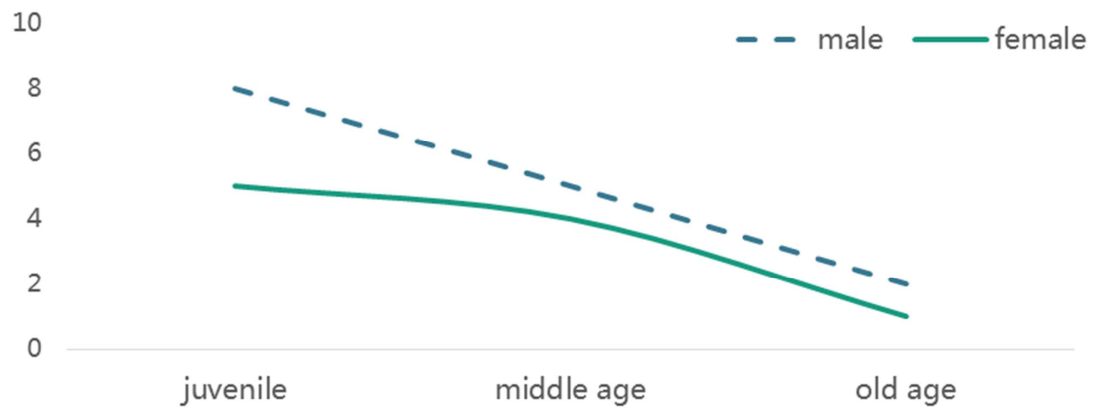

Figure 6. Age and gender curve of the status of coming out of the closet.

The constraints brought by gender and age of interviewees are often reflected in their coming out of the closet and self-identity. When we found this trend from the quantitative data, we added this question in the interview: Do you think your coming out of the closet has changed during your youth and middle age? But then we consider that such age changes are often accompanied by an important factor -- age. In the past 40 years of China's reform and opening up, the change of The Times means that the social environment has changed earthshaking. People's thinking has become more and more open. Many traditional dross have been gradually abandoned, and the society has become more open and inclusive. The researchers tried to use the SoC theory to explain the changing trend of individuals at different ages in self-identity and coming out of the closet:
Coming out of the closet at a young age often comes at no cost. Young people have nothing to lose in their happiness, hatred and passion. It is a kind of behavior without considering the consequences. As people move into middle age, the pressure on them to behave more socially, to hold down their jobs and to be less individualistic is increasing, not just from the pressure to pass on their children, but from their families and older generations. When people enter old age, both sexual desire and personality gradually decline, and the only thing that can improve is the moral requirements of the elderly. At this time, the elderly are no longer healthy and strong enough to bear a heavier psychological pressure, so they have to live their whole life in such loneliness and cover.

From the perspective of bibliometrics, we sorted out all the 
studies on gays and lesbians, college students/elderly gays on CNKI, and found the following trends:

Studies on gay men were significantly higher than on lesbians. There are significantly more studies on college students than older gay people. We can infer the reverse from the availability of the experimental study that lesbians and older gay people are more likely to hide their sexual orientation and tend to be neglected by society. In the study of bisexuality, there is little analysis of specific biological sex.

Table 11. CNKI Research Adjustment Table of Homosexuality.

\begin{tabular}{|c|c|c|c|c|}
\hline Search content & & Theme & Keywords & Article \\
\hline \multirow{5}{*}{ Homosexual } & Gay & 1099 & 293 & 278 \\
\hline & lesbian & 587 & 245 & 137 \\
\hline & College Student Homosexuality & 191 & 6 & 151 \\
\hline & Senile homosexuality & 1 & 0 & 0 \\
\hline & Homosexual & 6094 & 1613 & 1963 \\
\hline \multirow[b]{2}{*}{ bisexual } & Male bisexuality & 5 & 0 & 3 \\
\hline & Female bisexuality & 0 & 0 & 0 \\
\hline
\end{tabular}

The author searched the Chinese video site Bilibili for [50 questions], a popular online trend for gay couples to ask about their lives, and found that only six of the top 100 questions were posted by lesbians. It also suggests that more gay men may be more comfortable with social disclosure and experience less pressure when they come out. In addition, the popular beauty and male worship may also play a role, that is there is a culture of heterosexual women to respect gay men, and some studies speculate that the reason may be that the male role of women satisfies the fantasy of male status reduction and substitutes for suppression.

\subsubsection{Coming out Results from Approval, Results in \\ Uncertain}

Exposing one's sexual orientation often means troubles that follow, such as ridicule, hostility, ostracism, loss of friendship, and even breakdown of social relations. In the families, conflicts may be more intense, parents tend to follow mainstream views on the future of marriage and childbirth. When the children come out, on the one hand, it disrupts the long-term deployment of parents for them; on the other hand, it also brings great pressure to parents brought by their friends and relatives. Chinese parents are more lacking in understanding of homosexuality. Not only are they incomprehensible to minorities, but their doubts about sexual minorities are also accompanied by anxiety that their children are isolated and have no offspring and cannot have a stable old age. Parents face much more serious problem than we thought, just as they paid for us.

Most homosexuals have certain psychological preparation before coming out. After completing their self-identification, they will begin to make psychological presuppositions, guessing the scene after coming out and making preparations for it. The higher the individual's sense of self-identity, the easier it is to express themselves clearly and firmly, and the results of the behavior of coming out will in turn affect the individual's self-identity. Once coming out of the closet with success, the individual will reach harmony with the surrounding social relations, thereby enhancing their self-identity-My decision is correct, I am not a weird guy, everyone supports me, and I also support myself. But not everyone is that lucky. Once they fail to come out (which is normal), the individual's original self-identity will be contradictory, may reduce the original self-identity, thereby reducing the subsequent behavior possibility-My family (friends) don't seem to like my homosexuality. Am I really wrong? Should I change?.

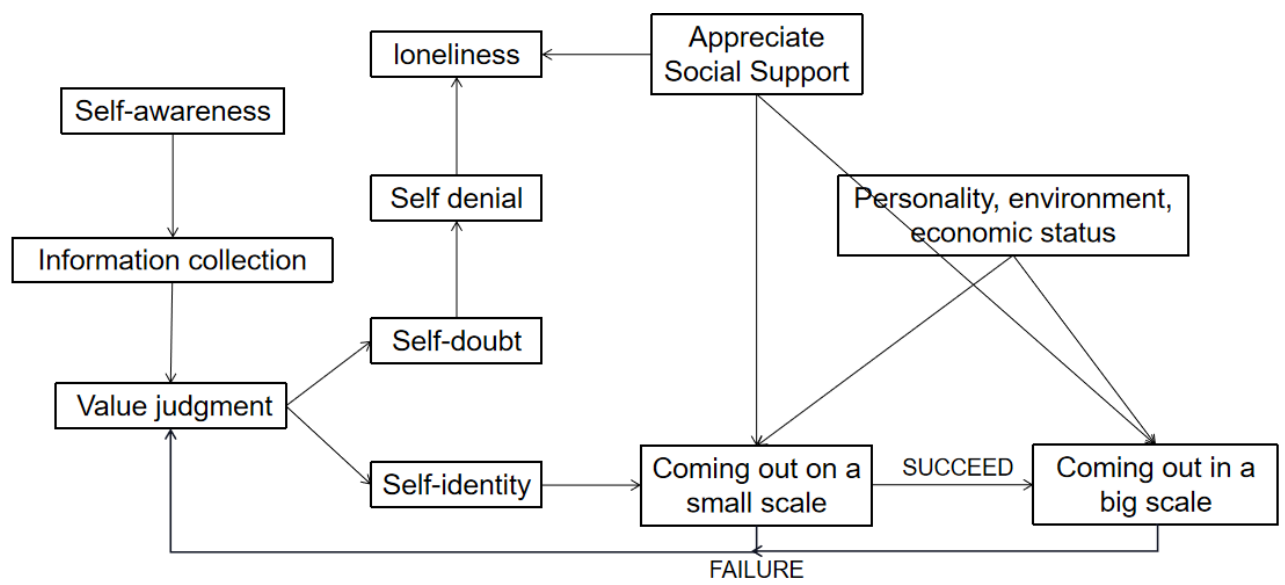

Figure 7. The model of coming out.

In our interviews, it can be clearly seen that the interviewee's self-identity with regard to sexual minorities is almost entirely established from the unknown. Perhaps due to the lack of sex education in China and the fact that the 
parents are secretive about sex, when they first discovered their sexual orientation, the interviewee would think that he was a rare monster. Typically, 521 mentioned At first I thought I was the only one on the planet. Actually it's not. There are many online. At the same time, almost all interviewees mentioned books, Internet and other resources, and the help given by the ones of the same kind on this difficult road. 211 said: I am better than the average person. I am the kind of person who can convince myself. When I saw gay resources (social media and other), there were already posts in forums and started to comfort everyone: We are not born a monster, homosexuality is common in Europe and America. Then I strengthened my identification with myself and searched for things in this area, so it is rare in China that I completed my self-identification very early and relatively smoothly. There is no period of discomfort or despair. I identified myself in junior high school. But keep the secret to the outside world defensively.

In the process of establishing self-identity, disidentification of one's own sexual minorities may come from the fear of detaching from traditional morality, mainstream norms and facing parental opposition, but identification is loyalty to and abiding by one's own desires. These internal sexual pleasure is so tempting and hard to refuse. 322 mentioned: It took $a$ long time to determine whether I am homo, I didn't read a book or anything. I feel comfortable in this way then go ahead. 121 said, The mood at that time, it was in junior high school, I didn't know much about this aspect, but had some contact. It may be that I don't think there is something, no contradictory. May be I had been exposed to some knowledge in this aspect, and I had seen other people's emotional experience, so there is no resisting emotions, just normal. But general environment gives a little restriction on oneself when expressing. Inevitably, human beings are social animals. Self-identity often comes not only from oneself, but also the perception of others and society's identification with us. Staying away from the mainstream, surrounded by the lack of the same kind and sex education and the imbalance of one's own desires, our interviewees stumbles along. Unfortunately, even if one have a high degree of self-identification, sexual minorities may still face the higher your self-identification, the stronger your loneliness. You are clearly aware that you are an alien, not accepted by everyone, lonely. 321 already has a stable physical and mental state: "This society may bring you a clearer peculiar feeling. Sexual minorities are like sheepdogs in a mixed herd, sheepdogs are good, but they are not sheep.

As an actual large group, exists chronically, the presence of sexual minorities is often reduced due to deliberate or unintentional neglect and suppression. For example, the US military's famous no question or no talk policy for military neutral minorities. Coming out is a means to break the transparent barrier that isolates one person from the surroundings. Exposing one's own sexual orientation to social connections, in the social atmosphere where minorities such as homosexuals and bisexuals have long been regarded as heterogeneous in China, undoubtedly requires courage.
Furthermore, many of them mentioned that only financial independence will support coming out. 211 expresses that economic independence and avoidance of negative circumstances is a conditions for choosing to come out: Not independent, you can't prove to others that you can live well. There is still a risk of others' acting in bad faith. But I think it doesn't matter after financial independence. I should avoid the environment that impedes me as much as possible, if I couldn't improve it, such as working for government.

Among the interviewees, most of them chose to hide until being forced to come out. It may be an accident, 211: It was almost the first year of high school that I had an lover, my mother found the search traces on the website. Then I closed room door, my parents cried and talked to me all the morning. May be caught by chance, 322 I was bumped by my dad when I was with my ex, then he told my mom, my parents were in divorce that time. It happened that they both wanted to change me, but he felt he couldn't, so they all showed a more enlightened attitude, but in fact they still feel that this is not good. 321 even affected by tragic campus violence: Probably in the second half of twelfth grade, I was forced to come out and then experienced campus violence and discrimination is, but there had been school violence before, then more serious. Including cold violence, even some verbal and excessive behavior. Even if they come out on their own initiative, it is rarely an exchange of life will based on trust, but a helpless vent. The bisexual person numbered 121 believes: Publicity results from depression. I'm depressed when I have no lover or sex. Born as crazy after depression, I choose to disclose it to some. As for hiding, it is to avoid quarrels and disputes and leave a way for my life.

It is worth noting that whether it is passive or active coming out, the results may not be satisfactory. 211: My parents cried and talked to me all morning. Because my parents can't accept it, they can only ask someone in the family to help. Anyway, both sides are feigning ignorance. The feedback under these circumstances undoubtedly mean that coming out is not a happy ending. After coming out, many people still pin their hopes on going out. 211's plan to reveal his love is pinned after going abroad: Go abroad and come out to find someone next year. 521 also said that although there is hope, I am currently planning to immigrate to Canada. After all, legal protection is very important. The domestic environment is definitely improving, the legalization of same-sex marriage will happen sooner or later.

Regarding the gender, we have noticed that the coming out of gay men has been more valued. Regarding this point, 322 expressed his own observation: My parents think that girls and girls are just for fun, but gays are not the condition. When man finds a man, the whole family goes mad. At least they pays attention to this problem. The long-term feudal society regarded women as objects and vassals. Even now, there is still inertia, and the true wishes of women are disconsidered.

At the beginning, we assumed that people with higher self-identification are more likely to choose to come out. But in actual research we found that the situation is particularly 
complicated. We live in an era of advanced information. Interviewees mentioned that they found their own kind peer through various channels to establish a sexual minority self-identity. For example, I read a magazine and found that male sexuality interesting. Before fifth grade., I can be sure that I prefer women. I have been indulged in gay websites at junior high school then lost interest in women over time.", "Then I strengthen my identity and search for things in this area, so it is a rare thing that me, a Chinese boy, completed self-identification smoothly without uncomfortable or desperate period. I turned book 'My Angel My Love' in the bookstore, and understood my feelings for her. Early, I thought I was the only one (homosexual). It turned out not, and there are many online. No big worries, at most I am worried that the one does not feel the same about me. 211 and 521 completed the sexual enlightenment through magazines and websites community to established self-identity.

The quantitative research and analysis of on the samples interviews show that people are more inclined to come out to people who are more intimate with them, from the perspective of the influence and interests involved in social relations. As a result, almost everyone comes out to close friends who are lowest interest related to them. In terms of family, children who receive more family support are more likely to come out. 322 mentioned: In my contact, people whose family relationships are harmonious are more likely to come out. And I know many T dressing up more neutral since they were young, like boys. Maybe their parents know it in their hearts, then accept it over time.

\subsubsection{Loneliness-an Enjoyable and Disgustful Feeling}

High-quality solitude is better than low-quality social interaction. In the previous review section, we analyze and explain the definition of loneliness: loneliness is an unpleasant experience of individuals when their social network is insufficient, including social relations insufficiency and low quality. The author reviews university life of myself. When in study or eating, I am alone. I am also thinking, am I really lonely? Being alone is the norm, but you can find someone to communicate when you need it. So lonely and alone are two different states. I am alone, but not lonely. I really enjoy the undisturbed state of doing things alone, but it does exist in the intricate social relations and has many connections. These connections are not uninterrupted, but are only connected when needed. This way makes me happy.

The interviewees' feelings and understanding gave us a glimpse of loneliness. 222 shared a lot of her own stories: After work, buy one and get second one half off makes me so lonely when one person finishes eating... For example, I have been molested as a child and remembered for ten years. For instance, questioning myself whether I am a waste. Not knowing how to throw the pot, I feel that everything is my responsibility. Such as, in fact, at my current stage, there is nothing at home that can help me, I can only rely on myself. 321 shared his story from the perspective of intimacy: The death of my grandpa... My girlfriend is at school, and disconnected. At that time, huge sense of loneliness surrounds me, I feel I have no contact with the world. 211 thinks: The difficulty degree of gay looking for male companions is more than ten times higher than normal men and women, loneliness is really a compulsory course. However, men generally have higher independence, so sometimes they also enjoy such undisturbed moments.

This kind of loneliness may come from the lack of intimacy, the departure of relatives, the feeling of helplessness in life and work situations, and the breakdown of family relationships. The essence is still the frustrating subjective feeling caused by the lack of part or all of the social network.

\subsubsection{Researchers'Self-report}

Among the methods of psychological research, there is a self-report method, which is generally considered to be subjective, and is generally used for case studies or some exploratory research. As a gay man, the author of this article has made in-depth thoughts on the four main variables of the study and the relationship between them, based on his own experience during the research process. Although there are personal subjective factors, it can still provide richer connotations and materials for this research.

The four variables studied in this article mainly include self-identity, perceived social support, coming out and loneliness. The four variables influence and blend with each other. In this study, there is no clear causal relationship. However, from the perspective of general logical reasoning, we believe that self-identification and perceived social support are independent variables, and the situation of coming out and loneliness are more biased to the dependent variable. The four variables all have unique characteristics. First, self-identification is an individual's cognition of oneself and value judgment process. However, comprehending social support as a subjective feeling that is dependent, has a destination, allowable subjective feeling is actually an external drive and an interpretation of the drive. Coming out is a behavioral manifestation. It is not only an expression of one's own sexual orientation but also a comprehensive result of self-identification, economic status, family relations and other factors. Loneliness is a negative emotional experience and feeling. From the simplest point, these 4 variables should have obvious positivity and negativity, but in real world, they are not as simple as we understand, rather complicated.

Among the scales used in this article the dimension of social support are family support, friend support, and other support. This scale has certain characteristics of the era, we think a new dimension can be added-network support. As a new form of media, the Internet plays an irreplaceable role in individual social relations. Netizens have become another social group no less than actual friends. In particular, it is an obscure behavior to disclose one's sexual orientation among homosexual groups, and the anonymous nature of the Internet happens to meet the needs s. Based on personal experience, not to mention openly expressing one's sexual 
orientation to the outside world, even within the homosexual group, there may be behaviors that hide one's sexual orientation. Because in the Chinese cultural background, the relationship between people strongly connected by a social network. Once one's sexual orientation is disclosed in an insecure situation, it may not only threaten intimacy, but also cause one's work and life troubled. Social support does increase the possibility of coming out to some extent, but the reason for coming out is not just social support. In addition, we also conducted a second discussion on the process of coming out.

Table 12. The process of coming out.

\begin{tabular}{ll}
\hline The extent of coming out & Concrete content \\
\hline Self-identification & Self-explore of individual \\
anonymous/in-group coming out & Coming out in a small area without strong loss \\
Affinity coming-out & Coming out in an intimate relationship that may produce certain contradictions \\
Public coming out & Coming out to the social relations around \\
Coming out manifestation & Advocating social movements with one's own homosexuality as a label \\
\hline
\end{tabular}

Last but not least to discuss is the sense of loneliness, which is also one of the many variables that puzzles me. The reason why I chose to study the role of loneliness in the gay community is that I am often alone in my life. I feel a little lonely, but it does not cause me distress or trouble. So I keep thinking about whether loneliness is a positive emotion or a negative emotion. From a positive perspective, I enjoy being alone. Being alone allows me to think calmly and observe the environment objectively. Many psychologists put the definition of loneliness on negative side, believing that loneliness will reduce the individual's mental health. So here I want to clarify the definition of the research. Loneliness is a state, an objective fact of working or living alone. And loneliness is the individual's feelings about this objective fact feeling negative. If the individual has different opinions on this objective fact, or has a positive psychological state, it cannot be called loneliness.

Therefore, the logic of our experimental design is when individuals pass through the collection of external information and their own value judgments, having a stronger self-identity, receiving a certain degree of social support, after meeting the objective conditions of economic conditions and opportunities, there may be behavior of coming out. When the individual's self-identity reaches a certain level, the heart in a more full state, there is no corresponding feeling of loneliness.

\section{Discussion}

This paper fails to classify and discuss the dimension of gender role in detail. Future research can focus on the measurement of gender role and analysis of its causes. Quantitative research can refer to the dimension of gender role obtained from qualitative research and give different weights to different dimensions.

One of the paradoxes in these results that we have long pondered is that women are significantly more likely to identify with themselves than men, but men are significantly more likely to come out of the closet than women. Some Chinese scholars also put forward the concept of identity without coming out of the closet. We analyzed this phenomenon and concluded that:

In terms of self-identity, although gender equality has become a basic national policy since the founding of the People's Republic of China, the society is still a male-dominated society in essence, and the maintenance of men's status still dominates the society. However, this has also become a kind of kidnapping and image constraint for male identity, that is, it requires men to be strong, dominant and masculine as the keynote. Once breaking through this image setting, they will be in a weak position. Similarly, women are also bound by their feminine and submissive images, and once their gender dimension is extended to men, the maintenance of male power from the society will also protect this kind of masculinity from women. For example, the female celebrities in history rarely have the image of delicate and weak attachment, more are the image of strong women - Wu Zetian, Hua Mulan, Li Qingzhao. Thus, one side of male homosexuality is degraded by men and one side is elevated by lesbians. The potential influence of this culture has an implicit influence on the attitudes of the public towards homosexuals of different genders, as well as the recognition and exploration of their own identity.

In terms of the situation of coming out of the closet, although men and women have been affected by the implicit influence of culture on their self-identity, the social tolerance towards men and the restriction towards women are more obvious. It is not uncommon for men to be dominant in sexual or intimate relationships, so men's behavior in intimate relationships is more likely to be tolerated. Male social and economic status of women are stronger than the more and more independent, more open, more tension on risk-taking, as a result, although in the process of self-identity is difficult, but once complete self-identification, less consider the external factors and more consideration to their inner true feelings and expressions.

If there is a physiological explanation, I would like to think about brain structure and hormone levels. The corpus callosum of males is significantly smaller than that of females, and their thinking is less disturbed by emotions and irrelevant factors in a short period of time. Therefore, there may be more sudden coming out of the closet. When they meet the right situation, there is no need to consider too many consequences. In terms of hormones, testosterone secreted by men is more likely to induce individual impulsive behavior and risky behavior. After all, coming out of the closet is 
indeed an impulsive risk.

A very important pair of variables in this study were self-identity and coming out of the closet, and we wanted to prove a causal relationship between them to some extent. The stronger the self-identity, the more likely it is to come out, but reading the literature and our findings suggest that there seems to be a necessary and insufficient relationship between them. We draw two distribution maps of self-identity and status of coming out, respectively. We find that the status of self-identity is an approximate normal distribution with high kurtosis and narrow. The status of coming out of the closet is a negative skewed distribution, that is, most people focus on coming out of the closet to a small extent.

Combined with the relationship model between coming-out of the closet and self-identity mentioned above, we have reason to believe that coming out of the closet lags behind self-identity to some extent. After a person has completed self-identity, he still needs some environmental factors, economic conditions, or even accidental events to come out of the closet. Coming-out of the closet is not an easy and pleasant thing. For homosexuals, it is no less than a gamble. They are gambling with their jobs, emotions and even social relations in the hope of gaining external recognition and acceptance.

In addition, there are also differences in sexual orientation, that is, homosexuals are more likely to come out than bisexuals. We concluded that the internal logic is (1) perceived sexual orientation discrimination against gay is greater than the pressure of bisexual sexual orientation discrimination of pressure, because bisexuals can avoid gay in order to avoid discrimination itself, but also caused out of difficulties, so gay is more like a no escape out (2) the bisexual on sexual orientation will suffer discrimination suspicion - feet trample two ship, which people think bisexuals may at the same time like two people of different gender, but in fact is not the case, often bisexual in order to avoid the truth of the suspicion may hide their orientation.

Although the network is so developed, social groups is increasingly close contact with the network, this paper still faces the possibility of a group of subjects sampling bias, those who are older or small group, and poor families can't through mobile phone or computer to log in groups and issues for network questionnaire and its bias group may not be involved.

In order to collect and express information on various aspects of people with different sexual orientation in more detail, more extensive and more in-depth, we applied two approaches -- quantitative and qualitative. The reason why we consider using two research methods is that most of the current international research on psychology focuses on quantitative research methods. Through quantitative research on certain psychological characteristics, the whole psychological process is digitized by using special scales or measuring instruments. However, not all processes can be processed into data. There are still some sensitive, perceptual and hidden psychological characteristics that cannot be directly detected, and they need to be projected and expressed in some linguistic and specific ways. However, these two methods have their own defects. First comes from the quantitative study of deficiency, although psychologists from all over the world like to use Numbers to describe individuals or groups of mental state or psychological quality, but we still feel the changeable and deep psychological, simply described is take the liberty of using digital and machinery, so we need a more moderate implicative and contains some of the methods to express philosophical speculations, although these may be very strong subjectivity, but still can give enlightenment on readers and subsequent research. The second is the qualitative research method. Due to the epidemic reasons and policies in China, it is difficult for us to have a face-to-face conversation, so we used telephone interview. However, we lost the opportunity to communicate with the interviewees face to face and observe their movements and expressions. The lack of information about the environment is extremely serious. We will also consider using field investigation or grounded theory to supplement the field data in subsequent studies.

In the interview, the groups willing to be interviewed are all individuals with a high sense of self-identity and can freely express their views on sexual orientation and other situations, but in fact, there are still many individuals in a confused, tangled and at a loss. We are not paying enough attention to these groups, we are not investing enough in sex education, it is still a hard day for each of them.

During the study, we found that although the data and results were significant, the effect value did not reach the ideal state, and we also thought about this phenomenon. First of all, we should rethink its reflections on this reflection, we have an inertial thinking, and no significant data can never be out of the report, not significant, the effect of value is not high data is of little value, but these data can still prove that we research idea from the side and elicitation for follow-up study provides a certain direction. Secondly, considering that this data collection was conducted on the Internet, the data collection method may not be relatively rigorous. Thirdly, when we construct the theory, we do not fully consider the relationship between various variables, but simply assume the co-variation relationship or correlation relationship. Finally, in this study, we use the intermediary effect and adjust the effect, although they can to some extent for some phenomenon of psychological quality and to make predictions, but they do not represent an absolute sense of cause and effect, because we didn't control under the condition of experiments, there is no change in time for their article. Because of our limited research funding and energy, and because of the epidemic, we were unable to carry out such an experiment.

As mentioned above, we also consider the disadvantages of conducting research on the Internet. Overall, we still believe that online survey is the most appropriate method available to us at present. The group we studied is not widely accepted in this society, so it is not possible to use the form of face-to-face or even street questionnaires in reality. The anonymity of the Internet well protects the privacy of these subjects, and at the same time makes the contents they fill in more real. 
In general questionnaires, people are faced with tendencies towards social approval and political correctness. They want to be regarded as excellent by others, as belonging to the majority, and as being recognized. At this point, we find a very different phenomenon in the online world. The so-called political correctness should mean that in a certain time and space environment, the content expressed by an individual is the tendency stipulated or established by the whole group ecology. We find it politically correct to support homosexuality in some parts of cyberspace, but only in an anonymous, open and inclusive environment. Therefore, the use of such questionnaires on the Internet can ensure the authenticity of the contents of the questionnaire filled by the subjects to a greater extent.

In addition, we want to make an ethical aspects of this paper suggests that we all are anonymous questionnaire to collect, even the researchers don't know these subjects identity is the most important personal information, related data in electronic form by us to the sharing of Baiduyunpan, will permanently sealed after the end of the study, unless there is a sequential testing necessary to use these data. We recorded all the interviewees, and these recording files will be encrypted and stored separately, and the personal information will be removed.

\section{Conclusion}

The research uses quantitative and qualitative methods to study the relationship between homosexuals' coming out, self-identity, loneliness and perceived social support in the context of traditional marriage concept as the mainstream ideology in China. In general, the results show that the level of self-identity of lesbians and bisexuals is significantly higher than that of men. Generally speaking, the higher the level of residence and education, the higher the level of self-identity. On the other hand, the higher the perceived social support of different groups, the lower their loneliness. Finally, in terms of coming out, young men tend to be the first group to come out, while older women tend to be the opposite.

\section{Acknowledgements}

This study was supported by Youth Innovation Team Development Plan in Shandong Province (2019) and Initial Scientific Research Fund for Ph.D. at Linyi University. We would like to express our gratitude for the support of this study.

\section{References}

[1] Ritch, C. \& Williams, S. (2016). Sexual Orientation: Categories or Continuum? Commentary on Bailey et al. Psychological Science in the Public Interest, 17 (2).

[2] Lamanna, M. A. et al. (2014) Marriages, Families, and Relationships: Making Choices in a Diverse Society. Cengage
Learning: 82 .

[3] Rosario, M.. et al, L. (2006). Sexual identity development among lesbian, gay, and bisexual youths: Consistency and change over time. Journal of Sex Research. 43 (1), 46-58.

[4] Li, Y. H. (2009). Homosexuality subculture. Hohhot: Inner Mongolia University Press: 24-65.

[5] Li, Z. (2006) Respect their world—Reading Li Yinhe's Homosexuality Subculture. Journal of Guangxi University for Nationalities, 28 (z2): 145-146, 166.

[6] Qazi, R. \&Glenn D. W. (2003). Born gay? The psychobiology of human sexual orientation. Personality and Individual Differences. 2003, 34 (8): 1337-82.

[7] Yu, W. \& Feng, T. J. Research progress on the biological causes of male homosexuality. Chinese Journal of Medical Genetics, 2012, 29 (2): 172-175.

[8] Yu, M. H. \& Wang, X. D. (2015) Research progress on the causes of male homosexuality. Chinese Sexual Sciences, (6): $119-122$.

[9] Wang X. J \& Wen J. H. (2002) Theoretical Discussion on the Causes of Homosexuality. Medicine and Philosophy, (4): 1-4.

[10] Wang, J. Y. et al. (2008) The biological causes of homosexuality. Guangzhou Medicine, (01): 44-46.

[11] Bai, X. H. (2008) The influence of the Internet on the way of homosexual interaction and identity. Jilin University.

[12] Wang Liyuan. A study on the self-identity of gay college students from the perspective of social work. Chinese Sexual Sciences, 2016, 25 (9): 139-143.

[13] Yang, L \& Xie, Y. J. (2011) A qualitative study on the development of homosexual men's self-identity. Chinese Sexual Sciences, 20 (05): 42-45+58.

[14] Cass, V. C. (1984). Homosexual Identity Formation: Testing a Theoretical Model. The Journal of Sex Research, 20 (2): 143 $-167$.

[15] Anthony R. D. (2002). Mental Health Problems among Lesbian, Gay, and Bisexual Youths Ages. Sage Publications, 7 (3). 14-21.

[16] Wang, Q. F. (2013). The evolution of the meaning of "coming out", theoretical explanation and empirical research. Academic Forum, 36 (01): 76-82.

[17] Wang, Q. F. (2011). Acknowledgement and not "coming out"-A survey of the living conditions of homosexuals. Journal of China Agricultural University (Social Science Edition), 28 (04): 142-153.

[18] Jing, J. et al. (2014). Coming out of homosexuality and the entanglement of family standard. Youth Studies (05): $79-86+96$

[19] Barry M. D. (1971). Coming out in the Gay World. Psychiatry, 34 (2), 180-197.

[20] Troiden, R. R. (1989). The Formation of Homosexual Identities. Journal of Homosexuality, 17 (1-2), 43-74.

[21] Plummer, K. (1995) Telling Sexual Stories, London: Routledge. 
[22] Taylor, B. (1999). Coming out' as a life transition: homosexual identity formation and its implications for health care practice. Journal of Advanced Nursing. 30 (2), 520-525.

[23] Greene, B. (1997). Ethnic and Cultural Diversity among Lesbians and Gay Men. London; Sage Publications, Inc.

[24] Dempsey, C. (1994). Health and Social lssues of Cay, Lesbian, and Bisexual Adolescents. Families in Society, 75 (3).

[25] Halley, J. E. (1988) Feminism, the Family and Politics of the Closet: Towards Equal Protection for Gay, Lesbian, and Bisexual Identity. UCLA Law Review, 36.

[26] Seidman, S. (2001). From Identity to Queer Politics. Citizenship Studies, 5 (3).

[27] Fu, X. X. (2012) Market Economy, Spatial Evolution and Sex: An Anthropological Observation of the Northeast Gay Group. (1980 2010). Open Times, (04): 133-149.

[28] Wei, W. (2007)"Piao Piao" in the city: the formation and changes of local homosexual identity in Chengdu. Society, (01): $67-97+207$

[29] Feng, J \& Zhao, N (2016). Homosexual Social Space and Social Networks in the Postmodern Geographical Context: Taking Beijing as an example. Acta Geographica Sinica, 71 (10): 1815-1832.

[30] Meyer, I. H. (2003), social stress, and mental health in lesbian, gay, and bisexual populations: conceptual issues and research evidence. Pub Med, 129 (5), 674-697.

[31] PlÖder M. et al (2014). Explaining the suicide risk. of sexual minority individuals by contrasting the minority stress model with suicide models. Pub Med, 43 (8), 2-37. Retrieved March 03, 2014, from http://dx.doi.org/10.1007/s10508-014-0268-4.

[32] Liu, R.. \& Mustanski, B. (2012). Suicidal Ideation and Self-Harm in Lesbian, Gay, Bisexual, and Transgender Youth. American Journal of Preventative Medicine, 42 (3), 221-228.

[33] Zhang, Y. et al. (2016). The impact of responsibility on homosexuals' life satisfaction: a moderated mediation effect [Jj]. Chinese Journal of Clinical Psychology, 2016, 24 (05): 921-924.

[34] Peplau, L. A. \& Cochran, S. D. (1981). Value Orientations in the Intimate Relationships of Gay Men. Journal of Homosexuality, 6 (3), 1-20.

[35] Li, C. A. (2003). An Intervention Study on the Loneliness of College Students. Journal of Shandong Youth Administrative Cadres College, (1): $46-48$.

[36] Da, H. M. \& Li, L. Q. (2009). A review of the research on loneliness of Chinese college students in the past ten years. Journal of Inner Mongolia Normal University (Education Science Edition), (1): 85-90.

[37] Wang, F. (2000) Analysis and countermeasures of contemporary youth's loneliness. Journal of Shandong Academy of Youth Management, (2): $10-11$

[38] Chen, F. G \& Chen, H. (2006) The status quo and prospects of contemporary youth loneliness research. Journal of Zibo Teachers College, (3): 20-22.

[39] Li, C. Y. \& Xu, Y. (1999). Review of loneliness psychology research. [J]. Social Psychology Research, (3): 14-16.
[40] Shou, A. Q. \& Chen, Y. Q. (2015) The relationship between online bullying, social support and loneliness among college students. Chinese Journal of Health Psychology: 233-237.

[41] Zhang. J. et al. (2015). Mental health of sexual minorities: theoretical models and research orientations. Advances in Psychological Science, 23 (6): 1021-1030.

[42] Hu, W. F. On the Mental Health of Homosexuality among College Students. Education Teaching Forum, (44): 194-195.

[43] $\mathrm{Hu}, \mathrm{J}$. S. et al. Loneliness, self-esteem and attachment of gay men.

[44] Wang, H. J. \& Tang, R. X. (2019). A Review of Researches on the Mental Health of Homosexuals in China. School Psychology, 17 (3): 201-204.

[45] Li, L. M. \& Zhao, B. H. The influence of homosexuals' public attitude perception on loneliness: the moderating effect of coming out. Chinese Journal of Clinical Psychology, 2015, 23 (5): 911-914.

[46] Ye, J. J. Research on the Influencing Factors of Undergraduates' Perception of Social Support. Psychological Science, 2005 (06): 190-193.

[47] Zhang, S. P. et al. (2015). A meta-analysis of understanding gender differences in social support. Psychological Development and Education, 31 (04): 393-401.

[48] Almeida, J. et al. (2009). Emotional distress among LGBT youth: The influence of perceived discrimination based on sexual orientation. Journal of youth and adolescence, 38 (7), 1001-1014.

[49] Bailey, J. M. et al. (2016). Sexual Orientation, Controversy, and Science. Psychological Science in the Public Interest. 17 (2), 45-101.

[50] Carroll, J. L. (2015). Sexuality Now: Embracing Diversity. Cengage Learning. 2015: 322. ISBN 1305446038.

[51] Chakraborty, A. et al. (2011). Mental health of the non-heterosexual population of England. Journal of Psychiatry, 198, 143-148.

[52] Denizet L. B. (2014). The scientific quest to prove that bisexuality exists. New York Times Magazine. Retrieved from https://www.nytimes.com/2014/03/23/magazine/the-scientificquest-to-prove-bisexuality-exists.htm/.

[53] Huang, F. R. et al (2010). Investigations on social psychology and sexual behavior of 52 male homosexual adolescents in Shanghai, 30 (5), 581-592.

[54] Hudson, S, N. \& Metcalf, H. (July). Inequality among lesbian, gay bisexual and transgender groups in the UK: a review of evidence. London: National Institution of Economic and Social Research.

[55] Jiang, H. B. (2008). Research on the relationship between college students' social support and loneliness. Fujian Normal University Fuqing Branch School News (5): 98-100.

[56] Chen, X. W. (2011). The relationship among college students' social support, social anxiety and loneliness. Heilongjiang Higher Education Research, (5): 121-123.

[57] Bian, C. (2018). Research on the relationship between male homosexual anxiety, depression and social support. Capital University of Economics and Business. 
[58] Li, L. M. \& Zhao, B. H. (2016). Research on the relationship between homosexuals' public attitude perception, social support and loneliness. Chinese Sexual Sciences, 25 (10): 141-143.

[59] Lin, Ji \& Tu, W. (2015). The relationship between college students' positive psychological qualities, coping styles, and social support. Chinese Journal of Health Psychology, 23 (02): 225-228.

[60] Zhou, S. X. (2013). The relationship between social support and real well-being: the mediating role of hope. Chinese Journal of Clinical Psychology, 21 (03): 515-517+499.

[61] Wu, S. S. (2013). Comprehend the relationship between social support, psychological capital and college students' academic achievement. Shandong Normal University.

[62] Zhang, Z. T. et al. (2012). The relationship between college students' parental rearing styles, social support, loneliness and subjective well-being. Chinese Journal of Health Psychology, 20 (07): 1080-1083.
[63] Shen, J. D. (2012). Research on the relationship between social support and well-being of college student. Soochow University.

[64] Chen, X. F. \& Shi, K. (2008). The impact of loneliness and social support on college students' mental health. Chinese Journal of Clinical Psychology, (05): 534-536.

[65] Xu, H. X. (2008). Understanding the influence of social support on college students' depression. Chinese Journal of Health Psychology, (04): 415-417.

[66] Quan H. Y. et al. (2008). The relationship between college students' mental health, personality, and social support [J]. Chinese Journal of Health Psychology, (02): 150-152.

[67] Quan H. Y. et al. (2007). Comprehend the role of social support in the regulation of personality and mental health. Chinese Journal of Clinical Psychology, (06): 598-600.

[68] Ye J. J. (2006). Comprehend social support, actual social support and college students' depression. Psychological Science, (05): 1141-1143+1131. 